\title{
Avaliação de propriedades físicas das madeiras de seis espécies florestais
}

\author{
Evaluation of physical properties of six \\ forest wood species
}

Henrique Römer Schulz ${ }^{1}$, Ezequiel Gallio ${ }^{1}$,
Andrey Pereira Acosta ${ }^{2}$, Darci Alberto Gatto ${ }^{2}$

\begin{abstract}
${ }^{1}$ UFPel - Universidade Federal de Pelotas, Centro de Desenvolvimento Tecnológico (CDTec), Rua Gomes Carneiro, n.1, CEP: 96010-610, Campus Anglo, Pelotas, Rio Grande do Sul, RS, Brasil.

${ }^{2}$ UFPel - Universidade Federal de Pelotas, Centro de Engenharias (CEng), Rua Benjamin Constant, CEP: 96010-020, Campus Cotada, Pelotas, Rio Grande do Sul, Brasil.

e-mail: henriqueschulz09@hotmail.com, egeng.florestal@gmail.com, andreysvp@gmail.com, darcigatto@yahoo.com
\end{abstract}

\section{RESUMO}

Este estudo tem como objetivo, avaliações e correlações entre propriedades físicas da madeira, sendo estas, massa especifica básica, teor de umidade, absorção de água, perda de massa em $50^{\circ} \mathrm{C}$ e $100^{\circ} \mathrm{C}$, contração linear máximo $(\beta)$, coeficiente de anisotropia de contração $(\mathrm{Ca} \beta)$, inchamento linear máximo $(\alpha)$ e coeficiente de anisotropia de inchamento $(\mathrm{Ca} \alpha)$, sendo analisado para as madeiras das seguintes espécies: Corymbia citriodora, Corymbia maculata, Eucalyptus dunnii, Eucalyptus grandis, Eucalyptus saligna e Eucalyptus tereticornis. Para isso, foram confeccionados corpos de prova de $20 \mathrm{~mm} \mathrm{x}$ $20 \mathrm{~mm} \times 15 \mathrm{~mm}$. Para as análises físicas, os corpos de prova foram submersos em água até a saturação, condicionados sob temperatura de $20^{\circ} \mathrm{C}$ com umidade relativa (UR) de $65 \%$ e secos em estufa $\left(100 \pm 3^{\circ} \mathrm{C}\right)$. Foram coletados dados de massa e dimensões desses corpos de prova em cada uma dessas etapas, para posterior cálculo das propriedades tecnológicas. Observou-se que as madeiras das sete espécies apresentaram diferença significativa. Analisando-se as propriedades de perda de massa a $100^{\circ} \mathrm{C}$ e massa específica básica, como também, anisotropia para inchamento (Ca $\left.\alpha\right)$ e contração (Ca $\left.\beta\right)$, apresentam uma correlação significativa, sendo diretamente proporcional. A massa específica básica e absorção de água, perda de massa a $100^{\circ} \mathrm{C}$ e absorção de água, Coeficiente de Anisotropia de Contração (Ca $\beta$ ) e absorção de água, apresentaram correlações significativas, sendo essas propriedades inversamente proporcionais. As madeiras do gênero Corymbia apresentaram valores médios de propriedades tecnológicas, superiores às do gênero Eucalyptus, destacando a madeira Corymbia citriodora.

Palavras-chave: Eucalyptus; Corymbia; massa específica básica, contração, inchamento.

\section{ABSTRACT}

This study aims at assessing and correlating physical properties of wood, such as basic specific mass, moisture content, water absorption, loss of mass at $50^{\circ} \mathrm{C}$ and $100^{\circ} \mathrm{C}$, maximum linear contraction $(\beta)$, coefficient of contraction anisotropy $(\mathrm{Ca} \beta)$, maximum linear swelling $(\alpha)$ and coefficient of swelling anisotropy $(\mathrm{Ca} \alpha)$, being analyzed for wood of the following species: Corymbia citriodora, Corymbia maculata, Eucalyptus dunnii, Eucalyptus grandis, Eucalyptus saligna and Eucalyptus tereticornis. For that, $20 \mathrm{~mm}$ x $20 \mathrm{~mm}$ x $15 \mathrm{~mm}$ specimens were prepared. For the physical analyzes, the specimens were submerged in water until saturated, conditioned at a temperature of $20^{\circ} \mathrm{C}$ with relative humidity (RH) of $65 \%$ and oven dried $\left(100 \pm 3^{\circ} \mathrm{C}\right)$. Mass data and dimensions of these specimens were collected in each of these stages, for later calculation of the technological properties. It was observed that the wood of the seven species presented significant difference. Analyzing the mass loss properties at $100^{\circ} \mathrm{C}$ and basic specific mass, as well as anisotropy for swelling $(\mathrm{Ca} \alpha)$ and contraction $(\mathrm{Ca} \beta)$, they present a significant correlation, being directly proportional. The basic specific mass and water absorption, loss of mass at $100^{\circ} \mathrm{C}$ and water absorption, Coefficient of Contraction Anisotropy $(\mathrm{Ca} \beta)$ and water absorption, showed significant correlations, being these inversely proportional properties. The wood of the genus Corymbia presented average values of technological properties superior to those of the genus Eucalyptus, highlighting the wood Corymbia citriodora. 
Keywords: Eucalyptus; Corymbia; basic specific gravity, contraction, swelling.

\section{INTRODUÇÃO}

Dentre as florestas plantadas que fornecem matéria prima para o setor madeireiro nacional, o gênero Eucalyptus recebe grande destaque [1]. Essa importância está relacionada pela facilidade de implantação em grandes maciços, versatilidade de aplicação de sua madeira, devido ao grande número de espécies dentro desse gênero e à velocidade de desenvolvimento de suas árvores.

Conforme o IBÁ [2], analisando o gênero de Eucalyptus o Brasil possuía uma área de aproximadamente 5,6 milhões de hectares plantados no país. A grande demanda do setor por esse gênero pode ser atribuída a qualidade da sua madeira e a versatilidade de utilizações.

Contudo, a qualidade desse material está diretamente relacionada as suas características tecnológicas, compostas estas por meio das propriedades físicas, químicas, mecânicas, térmicas, elétricas e acústicas. De acordo com Bowyer et. al [3], como propriedades físicas pode-se destacar a massa especifica básica, retratibilidade e teor de umidade. A massa específica está ligada a proporção de matéria lenhosa por unidade de volume, como também em relação aos espaços vazios existentes em uma amostra.

O parâmetro físico (retratibilidade) é responsável pela variação das dimensões das peças de madeira, ocorrendo quando há redução ou o aumento do teor de umidade abaixo do ponto de saturação das fibras [4]. Essas variações na umidade fazem com que ocorram defeitos devido a madeira ser um material higroscópico, ou seja, dependendo do ambiente ao qual a madeira está submetida, ela pode tanto perder como reter água, fazendo com que ocorram modificações dimensionais na mesma, afetando a qualidade do produto final.

Propriedades físicas como a massa específica e a retratibilidade influenciam diretamente as demais características tecnológicas da madeira. Essa última, provoca a incidência de defeitos nas peças de madeira, diminuindo a qualidade do produto final. Dias Junior et al. [5], afirmam, que essa propriedade é utilizada, juntamente com outras, para determinação da qualidade da madeira, empregando um melhor uso da mesma como matéria prima industrial, e também se constata correlações com outras propriedades tecnológicas.

Portanto, com o aumento do interesse na utilização, das mais diversas espécies do gênero Eucalyptus, para fins estruturais, madeira serrada, celulose e papel, entre outros, e para se ter um produto final com qualidade é necessário o conhecimento de suas propriedades tecnológicas, possibilitando uma aplicação segura para qual será destinado.

Sendo assim, o objetivo deste trabalho é determinar propriedades físicas da madeira, sendo elas, massa especifica básica, teor de umidade, absorção de água, perda de massa em $50^{\circ} \mathrm{C}$ e $100^{\circ} \mathrm{C}$, contração Linear Máximo $(\beta)$, coeficiente de Anisotropia de Contração (Ca $\beta$ ), inchamento Linear Máximo $(\alpha)$ e coeficiente de Anisotropia de Inchamento $(\mathrm{Ca} \alpha)$ para as espécies de madeira Corymbia citriodora, Corymbia maculata, Eucalyptus dunnii, Eucalyptus grandis, Eucalyptus saligna e Eucalyptus tereticornis.

\section{MATERIAIS E MÉTODOS}

\subsection{Seleção e Obtenção do Material}

O material utilizado neste estudo, foi fornecido pela indústria Celulose Riograndense - CMPC, localizada em Guaíba e outra parte pelo Laboratório de Propriedades Físicas e Mecânicas da madeira, do curso de Engenharia Industrial Madeireira, da Universidade Federal de Pelotas, RS, onde ocorreu as análises das propriedades tecnológicas.

Foram utilizadas as espécies Corymbia maculata, Corymbia citriodora, Eucalyptus dunnii, Eucalyptus grandis, Eucalyptus saligna e Eucalyptus tereticornis, com idade de 22 anos.

Depois de selecionadas as espécies florestais, foi feita a amostragem, seguindo a normatização American Society for Testing and Materials - ASTM D 5536 [6], sendo estes livres de quaisquer defeitos como empenos, nós, rachaduras, madeira danificada por agentes biodegradadores.

Posteriormente, das tábuas provenientes das espécies selecionadas, foram confeccionados os corpos de prova (CP): $20 \mathrm{~mm}$ x $20 \mathrm{~mm} 15 \mathrm{~mm}$ (radial x tangencial x longitudinal), para a determinação de propriedades físicas, sendo 36 amostras por ensaio, 6 para cada espécie. Depois de dimensionados, esses corpos de prova ficaram acondicionados em câmara climatizada (temperatura de $20^{\circ} \mathrm{C}$ e $65 \%$ de umidade relativa) até que atinjam massa constante e um teor de umidade de $12 \%$. 


\subsection{Amostras na condição seca}

Para certas análises as amostras tinham que estar nas condições de seca, ou seja, teor de umidade próximo de $0 \%$, para essa condição ser atendida as amostras foram colocadas na estufa de escala laboratorial (capacidade de 100 litros e temperatura máxima de $\left.300^{\circ} \mathrm{C}\right)$, primeiramente a $50^{\circ} \mathrm{C}\left( \pm 3^{\circ} \mathrm{C}\right)$ e alcançando a temperatura de $100^{\circ} \mathrm{C}\left( \pm 3^{\circ} \mathrm{C}\right)$ por um período de 24 horas em cada faixa de temperatura.

\subsection{Caracterização das Propriedades físicas}

\subsubsection{Massa especifica básica}

A massa específica básica foi determinada por meio da adaptação da normatização da American Society for Testing and Materials - ASTM D 2395 [7], com auxílio de um paquímetro digital (com resolução de 0,01 mm) e balança analítica (com resolução de $0,001 \mathrm{~g}$ ), sendo que a massa na amostra em estado seco e o volume no estado saturado. Mensurou-se as dimensões e massa dos corpos de prova e por meio da equação (1), caracterizou se a mesma.

$$
\mathrm{TU}_{\text {Equilibrio }}=\left(\frac{\mathrm{M}_{12 \%}-\mathrm{M}_{0}}{\mathrm{M}_{0}}\right) * 100
$$

Em que: $\mathrm{M}_{0}=$ massa seca em estufa a $100^{\circ} \mathrm{C}\left( \pm 3^{\circ} \mathrm{C}\right)(\mathrm{g}) ; \mathrm{V}_{\text {Saturado }}=$ volume com a amostra saturada $\left(\mathrm{cm}^{3}\right) ; \rho_{\text {Bás }}=$ massa especifica básica $\left(\mathrm{g} / \mathrm{cm}^{3}\right)$.

\subsubsection{Teor de Umidade de Equilíbrio}

O teor de umidade de equilíbrio foi mensurado, com auxílio de uma balança analítica (com resolução de 0,001g), utilizada a adaptação da normatização ASTM D 2395 [7]. Mensurou-se massa dos corpos de prova com teor de umidade de aproximadamente $12 \%$ e nas condições de amostras secas, por meio da equação (2), caracterizou se a mesma.

$$
\mathrm{TU}_{\text {Equilibrio }}=\left(\frac{\mathrm{M}_{12 \%}-\mathrm{M}_{0}}{\mathrm{M}_{0}}\right) * 100
$$

Em que Em que: $\mathrm{M}_{12 \%}=$ massa na condição de $12 \%$ de Teor de Umidade $(\mathrm{g}) ; \mathrm{M}_{0}=$ massa seca em estufa a $100^{\circ} \mathrm{C}( \pm$ $\left.3^{\circ} \mathrm{C}\right)(\mathrm{g}) ; \mathrm{TU} \mathrm{Equilibrio}=$ Teor de umidade de equilíbrio (\%).

\subsubsection{Absorção de água}

A absorção de água foi verificada seguindo a adaptação da normatização ASTM D 2395 [7], onde para a massa das amostras estar na condição saturada, utilizou-se um dessecador com uma quantidade de água afim das amostras ficarem submersas, foi acoplado uma bomba de vácuo, afim de aumentar a eficiência do processo, por um período de 5 horas. Com o auxílio de uma balança analítica (com resolução de $0,001 \mathrm{~g}$ ). Onde utilizou-se a massa das amostras saturadas e secas, sendo verificado por meio da equação (3):

$$
\mathrm{AA}_{\%}=\left(\frac{\mathrm{M}_{\text {Saturada }}-\mathrm{M}_{0}}{\mathrm{M}_{0}}\right) * 100
$$
de água (\%).

Em que: $\mathrm{M}_{\text {Saturada }}=$ massa na condição Saturada $(\mathrm{g}) ; \mathrm{M}_{0}=$ massa seca em estufa a $100^{\circ} \mathrm{C}\left( \pm 3^{\circ} \mathrm{C}\right)(\mathrm{g}) ; \mathrm{AA}_{\%}=\mathrm{Absorção}$

\subsubsection{Perda de massa}

A perda de massa foi analisada, por meio da normatização ASTM D 2395 [7], com o auxílio de uma balança analítica (com resolução de $0,001 \mathrm{~g}$ ), para $50^{\circ} \mathrm{C}$ Equação (4) e $100^{\circ} \mathrm{C}$ Equação (5), sendo essas temperaturas alcançadas em uma estufa de escala laboratorial.

$$
\begin{aligned}
\mathrm{PM}_{\%} & =\left(\frac{\mathrm{M}_{12 \%}-\mathrm{M}_{50^{\circ} \mathrm{C}}}{\mathrm{M}_{12 \%}}\right) * 100 \\
\mathrm{PM}_{\%} & =\left(\frac{\mathrm{M}_{12 \%}-\mathrm{M}_{100^{\circ} \mathrm{C}}}{\mathrm{M}_{12 \%}}\right) * 100
\end{aligned}
$$


Em que: $\mathrm{M}_{12 \%}=$ massa na condição de $12 \%$ de Teor de Umidade $(\mathrm{g}) ; \mathrm{M}_{50^{\circ} \mathrm{C}}=$ massa seca em estufa a $50^{\circ} \mathrm{C}\left( \pm 3^{\circ} \mathrm{C}\right)$; $\mathrm{M}_{100^{\circ} \mathrm{C}}=$ massa seca em estufa a $100^{\circ} \mathrm{C}\left( \pm 3^{\circ} \mathrm{C}\right)(\mathrm{g}) ; \mathrm{PM}_{\%}=$ Perda de massa (\%).

\subsubsection{Contração e inchamento lineares máximos}

Verificou-se a retratibilidade da madeira por meio da técnica normativa ASTM D143-94 [8], onde foi verificada a contração e inchamento lineares máximos nos planos anatômicos tangenciais e radiais. Para tanto, foi utilizando um paquímetro de precisão de $\pm 0,01 \mathrm{~mm}$ em amostras saturadas, onde foi medido as dimensões nos eixos radiais e tangenciais em seguida foram empregados os valores obtidos nas equações $6,7,8,9,10$ e 11 para a determinação da contração linear máxima (tangencial e radial), inchamento linear máximo (tangencial e radial), anisotropia para contração e inchamento, respectivamente.

$$
\begin{aligned}
& \beta_{\mathrm{T}}=\left(\frac{\mathrm{T}_{\text {Saturado }}-\mathrm{T}_{0}}{\mathrm{~T}_{\text {Saturado }}}\right) * 100 \\
& \beta_{\mathrm{R}}=\left(\frac{\mathrm{R}_{\text {Saturado }}-\mathrm{R}_{0}}{\mathrm{R}_{\text {Saturado }}}\right) * 100 \\
& (7) \\
& \alpha_{\mathrm{T}}=\left(\frac{\mathrm{T}_{\text {Saturado }}-\mathrm{T}_{0}}{\mathrm{~T}_{0}}\right) * 100 \\
& \alpha_{\mathrm{R}}=\left(\frac{\mathrm{R}_{\text {Saturado }}-\mathrm{R}_{0}}{\mathrm{R}_{0}}\right) * 100 \\
& \mathrm{Ca}_{\beta}=\left(\frac{\beta_{\mathrm{T}}}{\beta_{\mathrm{R}}}\right) \\
& \mathrm{Ca}_{\alpha}=\left(\frac{\alpha_{\mathrm{T}}}{\alpha_{\mathrm{R}}}\right)
\end{aligned}
$$

Em que: $\beta \mathrm{T}$ - contração linear máxima no plano anatômico tangencial (\%); $\beta \mathrm{R}$ - contração linear máxima no plano anatômico radial (\%); $\alpha_{\mathrm{T}}-$ inchamento linear máximo no plano anatômico tangencial (\%); $\alpha_{\mathrm{R}}$ - inchamento linear máximo no plano anatômico radial (\%); $\mathrm{T}_{\text {Saturado }}$ e $\mathrm{R}_{\text {Saturado }}$ - dimensões dos planos tangencial e radial em condição saturada de umidade (mm); $\mathrm{T}_{0}$ e $\mathrm{R}_{0}$ - dimensão dos planos tangencial e radial em condição seca à $103 \pm 2^{\circ} \mathrm{C}(\mathrm{mm})$; Caß $=$ Coeficiente de Anisotropia de Contração; $\mathrm{Ca} \alpha=$ Coeficiente de Anisotropia de inchamento.

\subsection{Análise estatística dos resultados das propriedades}

Primeiramente foram coletados os dados obtidos através dos testes físicos normatizados e posteriormente foram feitas análises descritivas, sendo estas: médias, desvio padrão, coeficiente de variação, sendo as médias comparadas, posteriormente, por meio do teste LSD de Fisher, com 5\% de probabilidade de erro e correlação de Pearson, para as propriedades de interesse.

\section{RESULTADOS E DISCUSSÃO}

São apresentados a análise descritiva apresentando os valores médios para as propriedades tecnológicas, massa especifica básica, teor de umidade de equilibro e absorção de água na Tabela 1. Pode-se observar que tais propriedades variam significativamente entre as espécies.

Tabela 1: Estatística descritiva para massa especifica básica, Teor de umidade de equilíbrio e Absorção de água.

\begin{tabular}{c|c|c|c|c|c|c}
\hline \multirow{2}{*}{ AMOSTRAS } & \multicolumn{2}{|c|}{$\begin{array}{c}\text { MASSA ESPECIFICA } \\
\text { BÁSICA (G/CM }\end{array}$} & \multicolumn{2}{c|}{$\begin{array}{c}\text { TEOR DE UMIDADE DE } \\
\text { EQUILÍBRIO (\%) }\end{array}$} & \multicolumn{2}{c}{ ABSORÇÃO DE ÁGUA (\%) } \\
\cline { 2 - 8 } & MÉDIA & CV & MÉDIA & CV & MÉDIA & CV \\
\hline Corymbia citriodora & $0,87 \mathrm{~F}(0,01)$ & 1,50 & $10,24 \mathrm{~A}(0,26)$ & 2,52 & $40,37 \mathrm{~A}(2,86)$ & 7,09 \\
\hline Corymbia maculata & $0,72 \mathrm{D}(0,01)$ & 0,71 & $10,06 \mathrm{~A}(0,26)$ & 2,62 & $57,52 \mathrm{C}(3,70)$ & 6,43 \\
\hline Eucalyptus dunnii & $0,55 \mathrm{C}(0,03)$ & 4,59 & $9,74 \mathrm{~A}(0,203)$ & 2,08 & $99,15 \mathrm{D}(9,74)$ & 9,82 \\
\hline
\end{tabular}




\begin{tabular}{c|c|c|c|c|c|c}
\hline Eucalyptus grandis & $0,50 \mathrm{~B}(0,01)$ & 0,92 & $9,95 \mathrm{~A}(0,20)$ & 2,03 & $96,37 \mathrm{D}(1,78)$ & 1,85 \\
\hline Eucalyptus saligna & $0,44 \mathrm{~A}(0,01)$ & 1,68 & $10,25 \mathrm{~A}(0,37)$ & 3,56 & $114,39 \mathrm{E}(5,87)$ & 5,13 \\
\hline Eucalyptus tereticornis & $0,77 \mathrm{E}(0,02)$ & 4,71 & $12,22 \mathrm{~B}(0,82)$ & 6,71 & $48,91 \mathrm{~B}(5,06)$ & 10,35 \\
\hline $\mathrm{F}$ & $363,76^{*}$ & - & $26,56 *$ & - & $190,87 *$ & - \\
\hline
\end{tabular}

Em que: Valores entre parênteses = Desvio Padrão na unidade da medida de cada propriedade; Letras diferentes, nas colunas, indicam diferenças significativas entre as espécies, conforme o teste LSD Fisher, em 95\% de confiança; CV = coeficiente de variação (\%); * - significativo em 5\% de probabilidade de erro, pelo teste $\mathrm{F}(\mathrm{p}<0,05)$.

Pode-se afirmar pelas médias observadas, dentre as seis espécies, que as madeiras de Corymbia citriodora e Eucalyptus saligna apresentaram o maior e o menor valor de massa especifica básica, sendo os mesmos de $0,87 \mathrm{~g} / \mathrm{cm}^{3}$ e 0,44 $\mathrm{g} / \mathrm{cm}^{3}$, respectivamente.

Segundo Veiga [9] ao estudar espécie de C. citriodora, constatou um valor de $0,71 \mathrm{~g} / \mathrm{cm}^{3}$ para densidade básica. Morais [10], observou um valor de densidade básica de $0,546 \mathrm{~g} / \mathrm{cm}^{3}$ para a espécie de Eucalyptus grandis. Rodrigues [11], obteve de massa específica a um teor de umidade de $12 \%$, para as espécies de Corymbia citriodora, Corymbia maculata, Eucalyptus saligna e Eucalyptus dunnii, os valores médios de $1,0 \mathrm{~g} / \mathrm{cm}^{3}, 0,93 \mathrm{~g} / \mathrm{cm}^{3}, 0,73 \mathrm{~g} / \mathrm{cm}^{3}, 0,69 \mathrm{~g} / \mathrm{cm}^{3}$, respectivamente. Trugilho et. al, [12] analisando espécies de Eucalyptus, estudando a propriedade de densidade básica para híbridos, constatou valores variando entre 0,446 a $0,590 \mathrm{~g} / \mathrm{cm}^{3}$.

Tais valores observados por diferentes autores são valores próximos ao encontrado nessa pesquisa. Essa variação na massa especifica pode ser explicada devido a madeira ser um material heterogêneo, portanto variando de espécies para espécie, em função da idade do material, posicionamento de onde foi coletado as amostras. Trevisan et al., [13], afirma que a massa específica é uma das propriedades que fornece informações diretas sobre as características da madeira, por estar relacionada com sua resistência e rigidez.

Para o teor de umidade de equilíbrio, a espécie de Eucalyptus tereticornis, obteve-se um valor de 12,22\% e para a espécie de Eucalyptus dunnii o valor de 9,74\%, sendo o maior valor e o menor valor, respectivamente. Segundo Gallio et al. [14], encontrou valores de teor de umidade de equilíbrio de 10,10\%, 10,70 \% e 10,70\% para as espécies de Corymbia maculata, Eucalyptus dunnii e Eucalyptus saligna, respectivamente.

Para a absorção de água a espécie de Eucalyptus saligna, obteve o maior valor, sendo de $114,39 \%$ e a Corymbia citriodora um valor de 40,37\%, sendo o menor quando comparado as outras espécies. Pode se notar uma diferença significativa no grupo amostral.

Pode-se observar que as espécies que apresentaram os menores valores médios de massa específica básica, foram as que apresentaram a maior absorção de água. De acordo com Clausen [15], conforme a maior quantidade de espaços vazios, ou seja, quanto maior a porosidade da madeira, maior será o fluxo de água em seu interior, o que ocasionara uma maior capacidade do material absorver água.

As propriedades tecnológicas, de perda de massa $(\%)$ a $50^{\circ} \mathrm{C}$ e $100^{\circ} \mathrm{C}$, em relação ao grupo controle é apresentado na Tabela 2. Pode-se observar que tais propriedades variam significativamente entre as espécies.

Tabela 2: Estatística descritiva para perda de massa de cada espécie.

\begin{tabular}{c|c|c|c|c}
\hline \multirow{2}{*}{ AMOSTRAS } & \multicolumn{2}{|c|}{ PERDA DE MASSA (\%) $-\mathbf{5 0} \mathbf{C}$} & \multicolumn{2}{c}{ PERDA DE MASSA (\%) $-\mathbf{1 0 0 ^ { \circ } \mathbf { C }}$} \\
\cline { 2 - 5 } & MÉDIA & $\mathbf{C V}$ & MÉDIA & CV \\
\hline Corymbia citriodora & $7,50 \mathrm{~A}(0,25)$ & 3,36 & $9,29 \mathrm{~A}(0,21)$ & 2,28 \\
\hline Corymbia maculata & $7,87 \mathrm{AB}(0,10)$ & 1,20 & $9,14 \mathrm{~A}(0,22)$ & 2,38 \\
\hline Eucalyptus dunnii & $7,76 \mathrm{AB}(0,08)$ & 0,98 & $8,87 \mathrm{~A}(0,17)$ & 1,90 \\
\hline Eucalyptus grandis & $7,85 \mathrm{AB}(0,23)$ & 2,86 & $9,05 \mathrm{~A}(0,17)$ & 1,84 \\
\hline Eucalyptus saligna & $7,98 \mathrm{~B}(0,29)$ & 3,67 & $9,29 \mathrm{~A}(0,30)$ & 3,24 \\
\hline Eucalyptus tereticornis & $9,15 \mathrm{C}(0,57)$ & 6,21 & $10,88 \mathrm{~B}(0,66)$ & 6,02 \\
\hline $\mathrm{F}$ & $19,97 *$ & - & $26,70 *$ & - \\
\hline
\end{tabular}

Em que: Valores entre parênteses = Desvio Padrão na unidade da medida de cada propriedade; Letras diferentes, nas colunas, indicam diferenças significativas entre as espécies, conforme o teste LSD Fisher, em 95\% de confiança; CV= coeficiente de variação $(\%)$; * significativo em $5 \%$ de probabilidade de erro, pelo teste $\mathrm{F}(\mathrm{p}<0,05)$. 
Pode-se afirmar pelas médias observadas, dentre as seis espécies, que as madeiras de Eucalyptus tereticornis apresentou o maior valor de perda de massa a $50^{\circ} \mathrm{C}$ e $100^{\circ} \mathrm{C}$, sendo de $9,15 \%$ e $10,88 \%$, essa perda de massa está ligada a água que é evaporada quando a madeira é submetida a calor.

A modificação térmica é um método em que a madeira é submetida a determinado ambiente em que é aplicado calor em distintas temperaturas, sendo primeiramente retirado água da madeira e posteriormente ocorrendo a degradação hemiceluloses, que são os mais sensíveis à ação do calor [16].

Para as propriedades tecnológicas, de contração no plano tangencial (\%), contração no plano radial (\%) e o coeficiente de anisotropia para contração são representados na Tabela 3. Pode-se observar que tais propriedades variam significativamente entre as espécies analisadas.

Tabela 3: Estatística descritiva para Contração Linear Máximo $(\beta)$ e Coeficiente de Anisotropia de Contração $(\mathrm{Ca} \beta)$, para os planos Tangenciais e Radiais.

\begin{tabular}{c|c|c|c|c|c|c}
\hline \multirow{2}{*}{ AMOSTRAS } & \multicolumn{2}{|c|}{$\beta$ - TANGENCIAL (\%) } & \multicolumn{2}{c|}{ B - RADIAL (\%) } & \multicolumn{2}{c}{ Ca $\beta$} \\
\cline { 2 - 7 } & MÉDIA & CV & MÉDIA & CV & MÉDIA & CV \\
\hline Corymbia citriodora & $8,56 \mathrm{C}(0,55)$ & 6,40 & $7,23 \mathrm{~B}(0,77)$ & 10,60 & $1,22 \mathrm{BC}(0,02)$ & 2,00 \\
\hline Corymbia maculata & $11,34 \mathrm{D}(0,73)$ & 6,40 & $8,41 \mathrm{C}(0,93)$ & 11,08 & $1,29 \mathrm{BC}(0,17)$ & 13,50 \\
\hline Eucalyptus dunnii & $11,09 \mathrm{D}(0,44)$ & 4,00 & $6,73 \mathrm{~B}(0,57)$ & 8,41 & $1,68 \mathrm{D}(0,18)$ & 10,45 \\
\hline Eucalyptus grandis & $7,80 \mathrm{~B}(0,25)$ & 3,20 & $6,90 \mathrm{~B}(0,56)$ & 8,16 & $1,14 \mathrm{AB}(0,07)$ & 6,31 \\
\hline Eucalyptus saligna & $6,74 \mathrm{~A}(0,66)$ & 9,75 & $6,72 \mathrm{~B}(0,97)$ & 14,49 & $1,03 \mathrm{~A}(0,23)$ & 21,91 \\
\hline Eucalyptus tereticornis & $7,21 \mathrm{AB}(0,09)$ & 1,24 & $5,10 \mathrm{~A}(0,23)$ & 4,56 & $1,38 \mathrm{C}(0,04)$ & 2,66 \\
\hline $\mathrm{F}$ & $73,12 *$ & - & $9,47 *$ & - & $14,51 *$ & - \\
\hline
\end{tabular}

Em que: Valores entre parênteses $=$ Desvio Padrão na unidade da medida de cada propriedade; Letras diferentes, nas colunas, indicam diferenças significativas entre as espécies, conforme o teste LSD Fisher, em 95\% de confiança; CV $=$ coeficiente de variação (\%); CV = coeficiente de variação (\%); $\beta$ - Tangencial = contração linear máxima no eixo tangencial, $\beta$ - Radial = contração linear máxima no eixo radial e Ca $\beta$ - coeficiente de anisotropia de contração; * - significativo em $5 \%$ de probabilidade de erro, pelo teste $\mathrm{F}(\mathrm{p}<0,05)$.

A madeira de Corymbia maculata foi a que apresentou maior valor de contração linear máximo tanto para o plano tangencial como radial, obtendo o valor de $11,34 \%$ e $8,97 \%$, respectivamente. A espécie de Eucalyptus saligna apresentou o menor valor de $6,74 \%$ para o plano tangencial e a espécie de Eucalyptus tereticornis apresentou o menor valor para o plano radial sendo de 5,08 \%. Em relação ao coeficiente de anisotropia para o plano tangencial, a espécie de Eucalyptus dunnii apresentou o maior valor, sendo de $1,68 \%$ e a espécie de Eucalyptus saligna o menor valor médio de 1,03\%.

Martins et al. [17], analisando a espécie de Corymbia maculata constataram uma contração no plano tangencial de $10,4 \%$ e radial $8,6 \%$, para o coeficiente de anisotropia de contração de 1,2, que são valores próximos aos encontrados nesse estudo. Segundo Oliveira et al. [4], para as espécies de Corymbia citriodora e Eucalyptus grandis, apresentaram valor de $10,0 \%$ e $10,1 \%$, para a retratibilidade para o plano tangencial. O mesmo autor constatou valore médios de coeficiente de anisotropia que variam de 1,4 a 2,1 para madeiras de espécies do gênero Eucalyptus e Corymbia.

De acordo Gallio et al. [14], encontrou valores de contração para o plano tangencial de $12,1 \%, 14,2 \%$, para o plano radial 9,7 \% e 9,4\% e fator de anisotropia de 1,2 e 1,6, para as espécies de Corymbia maculata e Eucalyptus dunnii, respectivamente. Analisando os dados em conjunto, os valores médios de contração linear máximo foram maiores para o plano tangencial em todas as espécies para esse estudo quando comparados ao plano radial.

Scanavaca Junior e Garcia [18], relatam que relação entre essas retrações gera o coeficiente anisotrópico, sendo a retração tangencial sempre maior que a radial, em que, quanto mais próximo de um for fator anisotrópico, mais alta é a qualidade da madeira, pois menor será a variação da sua movimentação em diferentes direções.

As propriedades tecnológicas, de inchamento no plano tangencial (\%), inchamento no plano radial (\%) e o coeficiente de anisotropia para inchamento são representadas na Tabela 4. Através da análise estatística pode-se observar que tais propriedades variam significativamente entre as espécies. 
Tabela 4: Estatística descritiva para Inchamento Linear Máximo $(\alpha)$ e Coeficiente de Anisotropia de Inchamento (Ca $\alpha)$, para os planos Tangenciais e Radiais.

\begin{tabular}{c|c|c|c|c|c|c}
\hline \multirow{2}{*}{ AMOSTRAS } & \multicolumn{2}{|c|}{$\alpha$ - TANGENCIAL $(\%)$} & \multicolumn{2}{|c|}{$\alpha$ - RADIAL (\%) } & \multicolumn{2}{c}{ Ca $\alpha$} \\
\cline { 2 - 7 } & MÉDIA & CV & MÉDIA & CV & MÉDIA & CV \\
\hline \multirow{2}{*}{ Corymbia citriodora } & $\begin{array}{c}9,37 \mathrm{~B} \\
(0,66)\end{array}$ & 7,01 & $7,80 \mathrm{~B}(0,90)$ & 11,48 & $1,24 \mathrm{ABC}(0,03)$ & $2,19 \%$ \\
\hline Corymbia maculata & $12,80 \mathrm{C}(0,93)$ & 7,24 & $9,19 \mathrm{C}(1,11)$ & 12,07 & $1,32 \mathrm{BC}(0,20)$ & $14,76 \%$ \\
\hline Eucalyptus dunnii & $12,47 \mathrm{C}(0,56)$ & 4,52 & $7,22 \mathrm{~B}(0,65)$ & 8,99 & $1,74 \mathrm{D}(0,08)$ & $4,43 \%$ \\
\hline Eucalyptus grandis & $8,47 \mathrm{~B}(0,29)$ & 3,47 & $7,41 \mathrm{~B}(0,65)$ & 8,77 & $1,15 \mathrm{AB}(0,08)$ & $6,76 \%$ \\
\hline Eucalyptus saligna & $7,23 \mathrm{~A}(0,75)$ & 10,41 & $7,21 \mathrm{~B}(1,12)$ & 15,54 & $1,03 \mathrm{~A}(0,24)$ & $23,38 \%$ \\
\hline Eucalyptus tereticornis & $7,76 \mathrm{~A}(0,10)$ & 1,33 & $5,37 \mathrm{~A}(0,26)$ & 4,80 & $1,41 \mathrm{C}(0,04)$ & $2,94 \%$ \\
\hline $\mathrm{F}$ & $72,65 *$ & - & $9,30 *$ & - & $13,68 *$ & - \\
\hline
\end{tabular}

Em que: Valores entre parênteses = Desvio Padrão na unidade da medida de cada propriedade; Letras diferentes, nas colunas, indicam diferenças significativas entre as espécies, conforme o teste LSD Fisher, em 95\% de confiança; CV = coeficiente de variação $(\%) ; \mathrm{CV}=$ coeficiente de variação $(\%)$; $\mathrm{N}^{\mathrm{o}}=$ número de repetições; $\alpha$ - Tangencial = inchamento linear máxima no eixo tangencial, $\alpha$ - Radial = inchamento linear máxima no eixo radial e Ca $\alpha$-coeficiente de anisotropia de inchamento; * - significativo em $5 \%$ de probabilidade de erro, pelo teste $\mathrm{F}(\mathrm{p}<0,05)$.

A madeira de Corymbia maculata foi a que apresentou maior valor de inchamento linear máximo tanto para o plano tangencial como radial, obtendo o valor de 12,80 \% e 9,89\%, respectivamente. A espécie de Eucalyptus saligna apresentou o menor valor de 7,23 \% para o plano tangencial e a espécie de Eucalyptus tereticornis apresentou o menor valor para o plano radial sendo de 5,36 \%. Em relação ao coeficiente de anisotropia para o plano tangencial, a espécie de Eucalyptus dunnii apresentou o maior valor, sendo de 1,77 \% e a espécie de Eucalyptus saligna o menor valor médio de 1,03\%.

Segundo Oliveira et al. [4], em seus estudos obteve como valores médios de retração radial para as espécies de Eucalyptus tereticornis (7,3\%), Corymbia citriodora (7,1\%) e Eucalyptus grandis (5,0\%). Logsdon et al. [19], relata que madeiras com anisotropia na faixa de 1,5 a 2,0 são consideradas enquanto normais, madeiras que apresentam coeficiente de anisotropia inferior a 1,5 são qualificadas como excelentes, por apresentarem elevada estabilidade dimensional.

Analisando a Tabela 5, apresenta-se a análise da correlação de Pearson, para cada espécie envolvida no estudo, analisando as propriedades de massa especifica básica, perda de massa a $100^{\circ} \mathrm{C}$, coeficiente de anisotropia tanto para inchamento, como para contração, assim como a absorção de água.

Tabela 5: Correlação de Pearson para as variáveis das espécies.

\begin{tabular}{c|c|c|c|c|c}
\hline & $\rho$ BÁS & $\begin{array}{c}\text { PERDA DE } \\
\text { MASSA 100 }\end{array}$ & CA $\boldsymbol{\alpha}$ & CA $\boldsymbol{\beta}$ & $\begin{array}{c}\text { ABSORÇÃO } \\
\text { DE ÁGUA }\end{array}$ \\
\hline$\rho$ BÁS & 1 & $0,4527^{*}$ & 0,3665 & 0,3779 & $-0,9793^{*}$ \\
\hline PERDA DE MASSA $\mathbf{1 0 0}^{\circ} \mathbf{C}$ & & 1 & 0,2289 & 0,2535 & $-0,4914^{*}$ \\
\hline $\mathbf{C A} \boldsymbol{C A} \boldsymbol{\beta}$ & & & 1 & $0,9994^{*}$ & $-0,3788$ \\
\hline ABSORÇÃO DE ÁGUA & & & & 1 & $-0,3915^{*}$ \\
\hline
\end{tabular}

Em que: * = significativo, teste feito com 95\% de confiança, onde mais próximo de 1, diretamente proporcional, e mais próximo de -1 , inversamente proporcional; $\rho$ BÁS $=$ massa específica básica; CA $\alpha=$ Anisotropia de inchamento; CA $\beta=$ Anisotropia de Contração.

A correlação de Pearson, indica se há ou não a correlação significativa entre propriedades estudadas, sendo diretamente proporcional próximo do 1 positivo e inversamente proporcional próximo de 1 negativo. Segundo Lira [20], a análise que permite verificar as associações ou relações, é nomeado como Análise de Correlação. Esse método verifica o grau de relacionamento entre as variáveis, fornecendo um número, indicando como as variáveis variam conjuntamente.

Analisando as propriedades de perda de massa a $100^{\circ} \mathrm{C}$ e massa específica básica, apresentam uma correlação significativa, sendo diretamente proporcional. Conforme a maior quantidade de massa, considerando uma mesma unidade de volume, maior será sua massa especifica básica, sendo assim o que pode se constatar, é que amostras com maior densidade 
básica e submetida a calor apresentarão uma tendência de maior perda de massa. O coeficiente de anisotropia para inchamento $(\mathrm{Ca} \alpha)$ e contração $(\mathrm{Ca} \beta)$, apresentou um valor significativo de 0,9994 , sendo diretamente proporcional.

A massa específica básica e absorção de água, perda de massa a $100^{\circ} \mathrm{C}$ e absorção de água, Coeficiente de Anisotropia de Contração $(\mathrm{Ca} \beta)$ e absorção de água, apresentaram correlações significativas, sendo essas propriedades inversamente proporcionais. Levando em consideração as correlações, a madeira que apresenta uma maior massa especifica básica, apresenta menor porosidade, e então uma menor capacidade de absorver água.

\section{CONCLUSÕES}

As madeiras do gênero Corymbia apresentaram valores médios de propriedades tecnológicas, superior as do gênero Eucalyptus, destacando para a maior massa especifica básica, o menor valor de absorção de água, valores que constatam boa estabilidade dimensional, sendo a espécie de Corymbia citriodora, recomendada a usos em ambientes externos, onde estará submetida a umidade.

Analisando as propriedades estudadas de diferentes espécies do gênero Eucalyptus e Corymbia, observou uma diferença significativa entre as mesmas, e é de grande importância o conhecimento sobre as mesmas para conseguir destinar sua matéria-prima para determinado processo, resultando em um produto final de extrema qualidade e com um alto rendimento.

Constata-se que certas propriedades estão correlacionadas com outras, sendo diretamente, perda de massa na maior temperatura analisada e massa específica básica. O coeficiente de anisotropia para inchamento (Ca $\alpha)$ e contração $(\mathrm{Ca} \beta)$, como também apresentando grandezas inversamente proporcionais, A massa específica básica e absorção de água, perda de massa a $100^{\circ} \mathrm{C}$ e absorção de água, Coeficiente de Anisotropia de Contração $(\mathrm{Ca} \beta)$ e absorção de água.

\section{BIBLIOGRAFIA}

[1] BATISTA, D. C., KLITZKE, R. J., SANTOS, C. V. T. "Densidade básica e retratibilidade da madeira de clones de três espécies Eucalyptus". Ciência Florestal, v.20, n. 4, pp. 665-674, 2010.

[2] IBÁ - INDÚSTRIA BRASILEIRA DE ÁRVORES. “Relatório Ibá 2017: ano base 2016”. pp. 64, 2017.

[3] BOWYER, J. L., SHMULSKY, R., HAYGREEN, J. G. "Forest products and wood science: an introduction". Ames Iowa:Iowa State Press, 4th ed. xiv, pp. 554, 2003.

[4] OLIVEIRA, J.T.S., FILHO, M.T., FIEDLER, N.C. “Avaliação da retratibilidade da madeira de sete espécies de Eucalyptus", Revista Árvore, v. 34, n.5, pp. 929-936, 2010.

[5] DIAS JUNIOR, A. F., DOS SANTOS, P. V., PACE, J. H. C., et al. "Caracterização da madeira de quatro espécies florestais para uso em movelaria”. Revista Ciência da Madeira, v.4, pp. 93-107, 2013.

[6] ASTM - AMERICAN SOCIETY FOR TESTING AND MATERIALS. D5536-94: "Standard practice for sampling forest trees for determination of clear wood properties", Philadelphia, 2010.

[7] ASTM - AMERICAN SOCIETY FOR TESTING AND MATERIALS. D2395-17. "Standard Test Methods for Density and Specific Gravity (Relative Density) of Wood and Wood-Based Materials”, Philadelphia, 2017.

[8] ASTM - AMERICAN SOCIETY FOR TESTING AND MATERIALS. D143-94: "Standard test methods for small clear specimens of timber", Philadelphia, 2014.

[9] VEIGA, T. R. L. A., LIMA, J. T., MONTEIRO, T. C., et al. "Propriedades mecânicas de amostras individualizadas da madeira e do carvão de Eucalyptus urophylla e de Corymbia citriodora". Scientias Forestalis, v. 46, n. 117, pp. 107-114, 2018.

[10] MORAIS, W. W. C., HASELEIN, C. R., SUSIN, F. et al. "Use of bambusa tuldoides and Eucalyptus grandis for manufacturing particleboards". Ciência Florestal, v. 28, n. 2, pp. 746-757, 2018.

[11] RODRIGUES, R. A. D. Variabilidade de propriedades físico-mecânicas em lotes de madeira serrada de eucalipto para a construção civil. Tese de D. Sc, USP, São Paulo, SP, Brasil, pp. 76, 2002.

[12] TRUGILHO, P. F., GOULART, S. L., ASSIS, A. O. et al. "Características de crescimento, composição química, física e estimativa de massa seca de madeira em clones e espécies de Eucalyptus jovens”. Ciência Rural, v. 45, n. 4, pp. 661-666, 2015.

[13] TREVISAN, R., HASELEIN, C.R.; MELO, R.R.; et al. "Variação radial da massa específica básica da madeira de Eucalyptus grandis W. Hill ex Maiden”. Floresta, n.3, pp. 553-559, 2008.

[14] GALLIO, E., ZANATTA, P., RIBES, D. D., et al. "Higroscopicidade da madeira de três espécies florestais deterioradas por térmitas Nasutitermes". Pesquisa Florestal Brasileira, v. 38, pp. 7, 2018. 
[15] CLAUSEN, C. A. "Biodeterioration of wood". Wood handbook: wood as an engineering material, v. 14 pp. 14.1$14.16,2010$.

[16] DUBEY, M. K., PANG, S., WALKER, J. “Changes in chemistry, color, dimensional stability and fungal resistance of Pinus radiata D. don wood with oil heattreatment”. Holzforschung, v. 66, n. 1, p. 49, 2012.

[17] MARTINS, M., SILVA, J. R. M. D., LIMA, J. T., et al. "Simulação em uso dos pisos de madeira de Eucalyptus sp e Corymbia maculata". Cerne, v. 19, n. 1, pp. 151-156, 2013.

[18] SCANAVACA JUNIOR, L; GARCIA, J.N. "Determinação das propriedades físicas e mecânicas da madeira de Eucalyptus urophylla”. Scientias Forestalis, n. 65, pp. 120-129, 2004.

[19] LOGSDON, N. B.; FINGER, Z.; ROSA, L. M. “Caracterização da madeira de Vochysia guianensis Aubl”. Engenharia Civil, n. 29, pp. 57-69, 2007.

[20] LIRA, S. A. Análise de correlação: abordagem teórica e de construção dos coeficientes com aplicações. Dissertação de M.Sc, UFPR, Curitiba, PR, Brasil, pp. 196, 2004.

\section{ORCID}

Henrique Römer Schulz

https://orcid.org/0000-0002-8162-7723

Ezequiel Gallio

https://orcid.org/0000-0002-0603-1065

Andrey Pereira Acosta

https://orcid.org/0000-0002-5074-3772

Darci Alberto Gatto

https://orcid.org/0000-0002-6805-3243 